\title{
Validade e precisão do Questionário de Relacionamento Central 6.0 (CRQ 6.0) para adultos com hepatite $\mathbf{C}$ crônica ${ }^{1}$
}

\author{
Gustavo Risso \\ Elisa Medici Pizão Yoshida² \\ Pontificia Universidade Católica de Campinas, Campinas-SP, Brasil
}

\begin{abstract}
Resumo: Portadores de hepatite $\mathrm{C}$ têm grande probabilidade de apresentar sintomas psicopatológicos e conflitos no relacionamento amoroso. Objetivou-se medir a precisão (consistência interna) e a validade (convergente e discriminante) do Questionário de Relacionamento Central 6.0 - CRQ 6.0, para adultos com hepatite C crônica. O CRQ 6.0, de autorrelato, avalia o padrão de relacionamento do indivíduo, segundo três componentes: Desejos (D), Respostas do Outro (RO), Respostas do $\mathrm{Eu}(\mathrm{RE})$. Participaram do estudo pacientes ambulatoriais com hepatite $\mathrm{C}$ crônica $(\mathrm{G} 1, \mathrm{n}=61)$ e acompanhantes de pacientes $(\mathrm{G} 2, \mathrm{n}=40)$. Todos responderam ao CRQ 6.0 e à Escala de Avaliação de Sintomas-40 (EAS-40). Os resultados indicaram consistência interna aceitável para os componentes do CRQ 6.0. Eles se correlacionaram significantemente com sintomas psicopatológicos pela EAS-40. Os escores do CRQ 6.0 foram significantemente mais elevados para participantes de G1, quando comparados aos de G2 (RO e RE), participantes sob medicação (RE) e mulheres (D e RE).
\end{abstract}

Palavras-chave: avaliação psicológica, escalas, psicologia da saúde, relações conjugais.

\section{Validity and reliability of the Central Relationship Questionnaire 6.0 (CRQ 6.0) for adults suffering from Chronic Hepatitis $\mathrm{C}$}

\begin{abstract}
People suffering from chronic hepatitis C have great probability of presenting psychopathologic symptoms and difficulties on relationships with romantic partners. This study aimed to estimate the internal consistency, convergent validity and discriminant validity of the Central Relationship Questionnaire 6.0 (CRQ 6.0) for adults with chronic hepatitis C. The CRQ 6.0 is a self-report, developed to assesses the individual's relationship patterns according to three components: Wishes (W), Responses from Others (RO), Responses of Self (RS). Participants were patients with chronic hepatitis C (G1, $\mathrm{n}=$ $61)$ and persons who accompanied patients $(\mathrm{G} 2, \mathrm{n}=40)$. They answered to the CRQ 6.0 and to the Symptoms Assessment Scale-40 (EAS-40). Results pointed to the acceptable internal consistency of the CRQ 6.0's components. They significantly correlated to psychopathological symptoms with EAS-40. Scores of the CRQ 6.0 were significantly higher for: participants of G1 (RO, RS), participants under medication (RS) and women (W, RS).
\end{abstract}

Keywords: psychological assessment, scaling (testing), health care psychology, marital relationship.

\section{Validez y fiabilidad de lo Cuestionario de Relación Central 6.0 (CRQ 6.0) para adultos con Hepatitis C Crónica}

\begin{abstract}
Resumen: Personas con hepatitis C crónica presentan alta probabilidad de sufrir de síntomas psicopatológicos y conflictos en las relaciones amorosas. El estudio tuvo como objetivo obtener pruebas de fiabilidad (consistencia interna), validad convergente y validad discriminante de lo Cuestionario de Relación Central 6.0 (CRQ 6,0) para adultos con hepatitis C crónica. El CRQ 6.0, de auto-informe, evalúa el patrón de relación del individuo en tres dimensiones: Deseos (D) Respuestas del Otro (RO), Respuestas del Self (RS). Participaran pacientes con hepatitis crónica $\mathrm{C}(\mathrm{G} 1, \mathrm{n}=61)$ y acompañantes de pacientes $(\mathrm{G} 2, \mathrm{n}=40)$. Todos han respondido al CRQ 6.0 y Escala de Evaluación de Síntomas-40 (EAS-40). El CRQ 6.0 mostró consistencia interna aceptable en sus componentes y correlaciones significantes con síntomas psicopatológicos pela EAS-40. Los escores de CRQ'60 fueran significantemente más altos para: participantes de G1 (RO, RS), participantes que tomaban medicación (RS), y para mujeres (D y RS).
\end{abstract}

Palabras clave: evaluación psicológica, escalas, psicología de la salud, relaciones conyugales.

A psicoterapia sofreu transformações importantes em seus modelos de intervenção nas últimas décadas, graças a um grande volume de pesquisas desenvolvidas nos

1 Apoio: CNPq. Este trabalho é derivado da Dissertação de Mestrado defendida pelo primeiro autor, sob a orientação da segunda autora, junto ao Programa de Pós-graduação Stricto Sensu em Psicologia da Pontifícia Universidade Católica de Campinas.

2 Endereço para correspondência:

Elisa Medici Pizão Yoshida. Pontifícia Universidade Católica de Campinas. Programa de Pós-graduação Stricto Sensu em Psicologia. Av. John Boyd Dunlop, $\mathrm{s} / \mathrm{n}^{\circ}$. Jd. Ipaussurama. CEP 13.060-904. Campinas SP, Brasil. E-mail: eyoshida.tln@terra.com.br anos de 1980 e 1990. Na busca de evidências empíricas que lhes dessem apoio, alguns desenvolvimentos metodológicos e ferramentas específicas foram propostos. Dentre eles, foram elaborados manuais de tratamento que traziam o detalhamento das intervenções preconizadas por uma determinada técnica psicoterápica e escalas de mensuração que permitiam avaliar em que medida a técnica estaria de fato sendo empregada pelos psicoterapeutas envolvidos em uma determinada pesquisa (Yoshida \& Rocha, 2007).

Descrito como um dos manuais mais utilizados nas abordagens psicodinâmicas, destaca-se o manual relativo à 
Psicoterapia Suportiva-Expressiva (Luborsky, 1984), fundada no pressuposto de que as principais dificuldades enfrentadas por pessoas adultas decorrem da persistência de padrões de relacionamentos originados na infância e cuja persistência resulta em conflitos na atualidade. Pela importância que têm para a dinâmica do paciente, são chamados de padrões centrais. Para cada indivíduo, um padrão central pode ser identificado a partir da forma como ele se relaciona com pessoas que têm importância afetiva em sua vida. O padrão central de relacionamento se refere, portanto, a uma forma característica que o indivíduo possui para se relacionar com os outros (Luborsky, 1984). A relação conflituosa inicia-se quando há uma diferença entre os padrões de relacionamento central entre duas ou mais pessoas, gerando um conflito entre desejos/impulsos e expectativas criadas que poderão não ser atendidas pelo outro (Barber, Foltz \& Weinryb, 1998; Luborsky, 1984; Luborsky \& Crits-Christoph, 1998).

A operacionalização desse construto deu origem ao desenvolvimento de um método denominado Core Conflictual Relationship Theme, mais conhecido pela sigla CCRT (Luborsky \& Crits-Christoph, 1998). O emprego do CCRT se dá por meio da identificação, nas transcrições de sessões de psicoterapias, do padrão de relacionamento conflituoso do paciente que se repete de forma mais ou menos constante em diferentes episódios do relacionamento, com pessoas importantes para ele. Em cada episódio, os componentes do padrão de relacionamento devem ser identificados: Desejos (D), Respostas do Outro (RO) e Respostas do Eu (RE). Os Ds, ROs, e REs predominantes configuram o CCRT do indivíduo (Luborsky \& Crits-Christoph, 1998; Rocha, 2004).

Apesar de ter se mostrado muito útil em pesquisas de resultado e de processos de psicoterapia, o CCRT depende da obtenção de acordo entre juízes independentes, devidamente treinados, o que toma muito tempo da equipe de pesquisa. Como consequência, investigações, que envolvem um grande número de participantes, ficam inviabilizadas (Barber \& cols. 1998; Weinryb, Barber, Foltz, Göransson \& Gustavsson, 2000). Como uma alternativa ao CCRT, foi construído o Questionário de Relacionamento Central (CRQ) (Barber \& cols., 1998), instrumento de autorrelato, com a finalidade de identificar o padrão central conflituoso presente no relacionamento afetivo/amoroso. Seus itens foram desenvolvidos com base em categorias padronizadas de avaliação do CCRT, para avaliar os três principais componentes: Desejos (D), Respostas do Outro (RO) e Respostas do Eu (RE). Inicialmente foram desenvolvidos 180 itens (65 para D; 51 para RO e 64 para RE) e suas propriedades psicométricas testadas junto a amostras de estudantes universitários $(n=315)$ e indivíduos da comunidade, convocados por meio de anúncios $(n=96)$. A análise fatorial exploratória revelou a existência de três componentes teoricamente distintos, sendo cada qual composto por subescalas que reproduziam de forma satisfatória as categorias de análise do CCRT. Ao final das análises, o componente $\mathrm{D}$ ficou constituído por 49 itens subdivididos em sete subescalas: ser suportivo, ser independente, estar em conflito, ser reconhecido, ser confiável, ser sexual e não ser abandonado. O RO ficou composto por 39 itens, também subdivididos em sete subescalas: me fere, me ama, é independente, me controla, é fora de controle, é ansioso, é sexual. E o componente RE, com 51 itens, ficou composto por oito subescalas: sinto-me valorizado (a), preocupo-me com o outro, sinto-me ansioso (a), sinto-me não querido (a), evito conflito, sou independente, sou sexual, sou dominador. As subescalas indicaram consistência interna satisfatória nos três componentes com Alfas $(\alpha \mathrm{s})$ de Cronbach, variando entre 0,78 e 0,95 no componente $\mathrm{D}(\alpha$ mediano $=0,90)$, entre 0,82 e 0,95 em RO $(\alpha$ mediano $=0,88)$ e entre 0,71 e $0,94 \mathrm{em} \mathrm{RE}$ $(\alpha$ mediano $=0,89)$. Além disso, o CRQ demonstrou evidências de validade convergente e divergente com instrumentos medindo problemas interpessoais - NEO - Five Factor Inventory e Inventory of Interpersonal Problems e sintomatologia - Beck Depression Inventory e Symptom Checklist90-Revised (Barber \& cols., 1998). Esta versão do CRQ foi posteriormente adaptada para o sueco e suas propriedades psicométricas reavaliadas, obtendo-se no componente $\mathrm{D} \mathrm{Al}$ fas de Cronbach entre 0,84 e 0,89, no RO entre 0,88 e 0,91 e no RE entre 0,82 e 0,89 (Weinryb \& cols., 2000).

Os resultados satisfatórios desses estudos ensejaram o desenvolvimento de novas versões, sempre no sentido de aprimorá-los (McCarthy, Gibbons \& Barber, 2008). Além disso, adaptações para populações e culturas distintas, tais como a chinesa, hebraica, alemã, sueca, espanhola, portuguesa e brasileira, já foram realizadas (Barber, Foltz, De Rubeis \& Landis, 2002), ainda que nem sempre acompanhadas de pesquisas de suas propriedades psicométricas. Rocha (2007) realizou uma primeira adaptação desse instrumento para o português do Brasil, tomando como base a versão 6.0 do CRQ (Barber, 1997). Esta versão foi posteriormente utilizada em pesquisas que tiveram como objetivo explorar as propriedades psicométricas do CRQ 6.0, junto a diferentes extratos da população brasileira: pacientes com diagnóstico positivo de HIV/AIDS (Massei \& Yoshida, 2009), pacientes com doenças coronarianas (Sanches, 2009) e mulheres vítimas de violência (Silva, 2008). A escolha desses extratos se deveu ao fato de se tratar de indivíduos que se encontram em situações que levam a sofrimentos psicológicos importantes e que podem ter interferência nos relacionamentos afetivos. Os resultados indicaram que a versão brasileira do CRQ detém consistência interna entre aceitável e satisfatória para os três componentes, com Alfas de Cronbach variando entre 0,80 e 0,85 no componente $\mathrm{D}$, entre 0,58 e 0,83 no RO e entre 0,59 e 0,81 no RE (Massei \& Yoshida, 2009, Sanches, 2009; Silva, 2008).

No presente estudo, foram testadas as propriedades psicométricas do CRQ 6.0 com amostra de portadores de hepatite $\mathrm{C}$ crônica. Examinou-se a consistência interna dos componentes do CRQ 6.0 e foram obtidas duas medidas de validade. Na primeira, validade convergente (Schutte \& Malouff, 1995), o CRQ 6.0 foi correlacionado com a Escala de Avaliação de Sintomas-40 (EAS-40) (Laloni, 2001), posto 
que há evidências na literatura que indicam que pessoas com hepatite $\mathrm{C}$ crônica apresentam vários sintomas e conflitos que atingem a esfera relacional (Blasiole, Shinkunas, LaBrecque, Arnold \& Zickmund, 2006). Na segunda, validade discriminante (Schutte \& Malouff, 1995), foram comparadas as avaliações do CRQ 6.0 de portadores de hepatite C crônica com as de pessoas sem diagnóstico de doença crônica.

\section{Hepatite $\mathrm{C}$ crônica e aspectos psicossociais}

A hepatite C crônica ou hepatite viral C (HVC) constitui um dos mais graves problemas de saúde médica e comunitária com que a população mundial tem se defrontado. Transmitida por via sexual ou transfusão de sangue, acreditase que o número de portadores seja muito maior do que o de indivíduos com HIV/AIDs (Ministério da Saúde, 2007). No Brasil, a prevalência média do vírus $\mathrm{C}$, em doadores de sangue, é cerca de $2 \%$. Em algumas regiões, no entanto, têm sido identificadas taxas maiores, como o Paraná e o Acre que apresentam prevalência próxima a $10 \%$. Estes dados permitem estimar, em pelo menos, três a quatro milhões de pessoas infectadas no país pelo vírus $\mathrm{C}$, ainda que não sejam oficiais. Ademais, essa doença possui um número grande de portadores que não sabe que a possui, devido ao início da doença ocorrer de forma assintomática (Ministério da Saúde, 2007; Teixeira, Martins-Filho \& Oliveira, 2005). Apesar da distribuição universal, sua prevalência varia com a localização geográfica, com os métodos diagnósticos empregados, com a seleção e a idade de cada grupo estudado. Alguns dados mostram uma maior prevalência em indivíduos com nível socioeconômico mais baixo e um leve predomínio do gênero masculino. Além disso, parceiros de pessoas infectadas possuem cerca de 3,7 vezes mais chances de serem infectados, se comparados com indivíduos da população em geral (Veronesi \& Focaccia, 2005).

A preocupação com a possibilidade de transtornos mentais e a redução na qualidade de vida, associada ao tratamento da HVC, têm levado profissionais da área médica e equipes interdisciplinares a solicitarem, rotineiramente, uma avaliação psicológica anterior ao tratamento. Principalmente nas condições crônicas, os processos patofisiológicos envolvidos e as adversidades neles implícitas podem afetar profundamente o funcionamento das pessoas em diversos níveis, tais como o emocional, social, vocacional e sexual (Miyazaki, Domingos, Valério, Souza, \& Silva, 2005). No que diz respeito à evolução da doença, apresenta uma fase aguda, na qual se encontram entre $10 \%$ e $15 \%$ dos portadores de HVC, estando os demais na fase crônica (Veronesi \& Focaccia, 2005). Além da condição ou situação de cronicidade ser em si mesma motivo de estresse psicológico, pode também ser fonte de novos estressores, tais como: a necessidade de mudanças no estilo de vida, a diminuição da energia física, alterações na aparência pessoal e as condições do próprio regime de tratamento. Como consequência, podem ser observados reações e/ou estados emocionais, entre os quais se incluem: insatisfação, insegurança, depressão, angústia, medo, sentimentos de desespero, fé, esperança, saudade e resignação. Fatores como isolamento social, preconceito e dificuldades de relacionamento também são citados, por causa dos efeitos colaterais físicos proporcionados pela doença (Miyazaki e cols., 2005).

Os motivos para um elevado índice de depressão em indivíduos infectados ainda são desconhecidos, embora dois fatores mereçam destaque: a HVC acomete predominantemente adultos jovens (prevalência mais elevada entre 30 e 40 anos de idade), o que pode elevar as preocupações sobre o futuro; e costuma estar associada a mais queixas somáticas do que as verificadas em pacientes com outros tipos de doença no fígado. (Thomson \& Finch, 2005). Há também uma grande preocupação com os efeitos colaterais psiquiátricos (estados alterados de humor, delírio, ansiedade e irritabilidade), provavelmente devidos ao uso do Interferon-alfa, um dos medicamentos indicados no tratamento da hepatite C, que parece estar relacionado à alteração do humor (Mulder e cols., 2000; Strader, Wright, Thomas \& Seef, 2004).

É preciso ressalvar que, apesar de doenças crônicas poderem ser vistas como um dos fatores mais estressantes na vida adulta, em alguns casos, proporcionam o estreitamento das relações afetivas, especialmente entre familiares e cônjuges. A aproximação ou reaproximação de pessoas relevantes para o paciente pode funcionar como suporte afetivo positivo e afetar, de forma adequada, os sistemas biológicos, comportamentos de saúde e o bem-estar destes pacientes (Abreu-Rodrigues \& Seidl, 2008). Em que medida essas situações fazem parte da regra ou das exceções, é, todavia, uma questão em aberto. As evidências empíricas têm, todavia, apontado, de forma mais consistente, o lado negativo das doenças crônicas que, conforme citado, incluem a associação de sintomas de ansiedade, depressão, prejuízos na qualidade de vida e raiva/hostilidade associados à medicação utilizada para o tratamento (Amodio e cols., 2005; Kraus, Schäfer, Faller, Csef, \& Scheurlen, 2003). A natureza dos conflitos psicológicos vivenciados pelos portadores de hepatite $\mathrm{C}$ segue, todavia, insuficientemente compreendida, havendo ainda grande carência de estudos sobre o impacto da doença nos relacionamentos familiares e com o parceiro amoroso (Blasiole e cols., 2006; Fabris e cols., 2006).

\section{Método}

\section{Participantes}

A amostra total foi composta por 101 participantes (49 mulheres e 52 homens), selecionados de forma não probabilística, em unidades ambulatoriais de hospital geral universitário, de cidade do interior do estado de São Paulo. Ficaram distribuídos em dois grupos: G1 - composto por 61 participantes com diagnóstico de hepatite $\mathrm{C}$ crônica (24 mulheres e 37 homens), com idades entre 26 e 71 anos (M=48,74 e $\mathrm{DP}=9,12)$; ensino fundamental $(72,13 \%) ; 70,5 \%$ eram casados, $55,74 \%$ estavam trabalhando, $93,44 \%$ possuíam alguma 
religião e $34,43 \%$ estavam em tratamento medicamentoso para HVC e G2 - composto por 40 acompanhantes de pacientes de outros ambulatórios do mesmo hospital universitário (25 mulheres e 15 homens), sem diagnóstico de doença crônica, com idades entre 18 e 54 anos $(\mathrm{M}=34$ e $\mathrm{DP}=11,10)$; ensino médio e superior (65\%); 65\% eram casados; $53,33 \%$ trabalhavam, 96,67\% tinham alguma religião, sem uso de medicação (100\%).

Comparando o G1 com o G2, é possível ver que o primeiro ficou composto, predominantemente, por homens (respectivamente, $60,66 \%$ e $37,50 \%$ ); com idades superiores a 40 anos $(81,96 \%$ vs. $32,50 \%)$ e com grau de escolaridade até o ensino fundamental $(72,31 \%$ vs. $35 \%)$ e cerca de um terço estava sob tratamento medicamentoso $(34,43 \%$ vs. $0 \%)$. Os grupos eram comparáveis quanto ao predomínio de casados $(70,5 \%$ vs. $65 \%)$, que trabalhavam $(55,74 \%$ vs. $53,33 \%)$ e com alguma religião $(93,44 \%$ vs. $96,67 \%)$.

\section{Instrumentos}

Questionário de Relacionamento Central 6.0 (CRQ 6.0) (Barber, 1997). A versão do CRQ utilizada foi traduzida e adaptada para o português do Brasil por Rocha (2007). Possui 101 itens divididos em três componentes: Desejos (D), Respostas do Outro (RO) e Respostas do Eu (RE). O componente D possui 40 itens divididos em 12 subescalas (ser fechado, ser distante, ser dominador, ser hostil, ser independente, ser amado, ser reconhecido, ser seguro, ser atraente, ser submisso, ser suportivo e ser de confiança); RO tem 23 itens e 8 subescalas (é distante, dominador, hostil, independente, amado, atraente, submisso e descontrolado); e o RE é formado por 38 itens e 13 subescalas (sou ambivalente, ansioso, fechado, distante, dominador, não sou querido, independente, não sou conflituoso, sou atraente, submisso, bem- sucedido, suportivo, valorizado). Há subescalas com valência positiva, e outras com valência negativa. As subescalas com valência positiva são compostas por itens com conotação afetiva positiva em relação ao parceiro. Itens negativos, tais como "eu gostaria de magoar o meu parceiro", têm valência negativa e precisam ter o escore invertido na avaliação do instrumento. São os seguintes os itens negativos: no componente D - 2, 6, 7, 8, 10, 14, 21, 24, 25, 33, 35,38 , 40; em RO: $3,4,6,7,9,11,12,14,16,17,18,20$, 21; e em RE: 4, 5, 6, 7, 10, 12, 13, 15, 16, 17, 19, 21, 23, $26,29,30,31,32,36,37$. A avaliação é obtida pela soma dos escores, sendo que quanto mais elevado o escore maior a probabilidade de conflito na subescala. Para responder ao CRQ 6.0, o indivíduo necessita ter tido um relacionamento com um parceiro, de pelo menos três meses, nos últimos três anos. Ele é solicitado a responder aos itens, referindo-se ao pior momento de seu relacionamento, e as respostas são dadas segundo uma escala Likert de 7 pontos na qual 1 corresponde a nunca e 7 , a sempre. Com relação ao intervalo possível, no componente $\mathrm{D}$, a intensidade do conflito varia entre 40 e 280; o componente RE, entre 23 e 161 e o RO, entre 38 e 266 .
Escala de Avaliação de Sintomas - EAS - 40 (Laloni, 2001). A EAS - 40 é uma escala de autorrelato, composta por 40 itens divididos em quatro dimensões: Psicoticismo, Obsessividade-Compulsividade, Somatização e Ansiedade. As respostas são fornecidas segundo uma escala Likert de três pontos que variam entre 0 (nenhum), 1 (pouco) e 2 (muito). A avaliação é feita pela estimativa da média aritmética dos escores de cada dimensão e obtenção do escore total, sendo que quanto maior o escore maior a severidade dos sintomas. A EAS-40 é uma adaptação da Symptom Checklist - Revised SCL-90-R (Derogatis, 1994) e validada para avaliar sintomas psicopatológicos na população hospitalar brasileira. Em estudo que teve por objetivo explorar o método de Jacobson e Truax, para a determinação de mudança clinicamente significante em sintomas avaliados por medidas de autorrelato, quando dados normativos não estão disponíveis, Yoshida (2008) encontrou, para as quatro dimensões da EAS-40, pontos de corte equivalentes a 1. Pesquisas realizadas no Brasil com a EAS-40 demonstraram índices satisfatórios com relação às suas propriedades psicométricas com pacientes clínicos internados em hospital geral (Laloni, 2001), com trabalhadores universitários e não universitários (Tombolato, 2005), pacientes com insuficiência renal (Pregnolato, 2005) e estudantes universitários (Yoshida \& Silva, 2007), entre outros.

\section{Procedimento de coleta de dados}

A coleta de dados do G1 foi realizada, de forma individual, logo após a consulta médica, no ambulatório de moléstias infecciosas onde os participantes recebiam atendimento. $\mathrm{O}$ encaminhamento para a pesquisa foi feito pelo médico responsável pelo setor, entre os pacientes que eram atendidos a cada dia de coleta. Para a composição de G2, recrutaram-se pessoas que acompanhavam pacientes de outros ambulatórios ou enfermarias, do mesmo hospital. Não se incluíram acompanhantes dos pacientes do ambulatório de doenças infecciosas, onde são atendidos os portadores de hepatite $C$, por se pressupor que eles vivenciariam os mesmos conflitos relacionais desses últimos, o que poderia exercer um viés sobre os resultados. Daí a opção por acompanhantes de pacientes de outras unidades. Além disso, como um procedimento de randomização das amostras mostrou-se inviável, a escolha de acompanhantes de pacientes do mesmo hospital teve como objetivo garantir certa homogeneidade no perfil sociodemográfico dos dois grupos amostrais. Os participantes foram abordados nas salas de espera dos ambulatórios e convidados a participar da pesquisa. A coleta de dados também foi feita individualmente, numa das salas do hospital. Adotaram-se, como critério de exclusão de G2, participantes com doenças crônicas tais como: diabetes, hipertensão, cardiopatias, entre outras. Em ambos os grupos, a participação foi voluntária, e a coleta de dados antecedida de Consentimento Livre e Esclarecido. A ordem de aplicação dos instrumentos foi alternada. O projeto de pesquisa foi aprovado pelo Comitê de Ética em Pesquisa da instituição, número do protocolo 247/08. 


\section{Procedimento de análise dos dados}

A avaliação da consistência interna dos itens de cada componente (D, RO e RE) foi realizada estimando-se os respectivos coeficientes Alfa de Cronbach $(\alpha)$. Esperavam-se resultados compatíveis com os evidenciados pela versão original do instrumento (Barber e cols., 1998). Para o primeiro estudo de validade convergente, a EAS-40 foi escolhida devido às qualidades psicométricas evidenciadas em amostras clínicas brasileiras, atendidas em hospitais gerais, como o da presente pesquisa (Laloni, 2001, Tombolato, 2005). Pressupunha-se que cada componente do CRQ 6.0 (D, RO e RE) apresentaria correlação significante $(\mathrm{p}<0,05)$ com cada dimensão da EAS40, assim como com o escore total da EAS-40. No segundo estudo de validade discriminante, a comparação dos escores do CRQ 6.0 de portadores de hepatite C crônica com os de pessoas sem diagnóstico de doenças crônicas, por meio da prova $\mathrm{U}$ de Mann Whitney, baseou-se na hipótese de que os primeiros apresentariam níveis mais elevados de conflito nos três componentes ( $\mathrm{D}, \mathrm{RO}$ e RE). Também eram esperados escores mais elevados para os participantes de G1 que se encontravam sob medicação, quando comparados aos sem medicação. E para os participantes de G1 do sexo feminino, quando comparados aos do sexo masculino.

\section{Resultados}

\section{Consistência Interna}

A Tabela 1 apresenta os valores do Alfa de Cronbach $(\alpha)$ dos três componentes do CRQ 6.0 (D, RO e RE) para a amostra total $(n=101)$, para o $G 1(n=61)$ e o $G 2(n=40)$. Além disso, apresenta o item com menor consistência dentro do componente e o valor do $\alpha$, caso o item seja retirado.

Tabela 1

Coeficiente Alfa de Cronbach ( $\alpha$ ) de cada componente do CRQ 6.0 para a amostra total, G1 e G2, item com menor consistência (IMC), correlação item-total e $\alpha$ (após retirado o item)

\begin{tabular}{|c|c|c|c|c|c|c|}
\hline Amostra & Componente & $\mathbf{N}^{\mathbf{0}}$ Itens & $\boldsymbol{\alpha}$ & IMC & Correlação com o Total ${ }^{(a)}$ & $\alpha$ (após retirado o item) \\
\hline \multirow[t]{3}{*}{ Total $(n=101)$} & $\mathrm{D}$ & 40 & 0,835 & D7 & -0.198 & 0,845 \\
\hline & RO & 23 & 0,842 & RO10 & -0.205 & 0,859 \\
\hline & $\mathrm{RE}$ & 38 & 0,860 & RS8 & -0.206 & 0,868 \\
\hline \multirow[t]{3}{*}{$\mathrm{G} 1(\mathrm{n}=61)$} & $\mathrm{D}$ & 40 & 0,858 & D7 & -0.371 & 0,870 \\
\hline & RO & 23 & 0,867 & RO10 & -0.272 & 0,883 \\
\hline & $\mathrm{RE}$ & 38 & 0,878 & RS19 & -0.154 & 0,885 \\
\hline \multirow[t]{3}{*}{$\mathrm{G} 2(\mathrm{n}=40)$} & $\mathrm{D}$ & 40 & 0,825 & D36 & -0.291 & 0,838 \\
\hline & RO & 23 & 0,808 & RO19 & -0.267 & 0,830 \\
\hline & $\mathrm{RE}$ & 38 & 0,837 & RS26 & -0.446 & 0,853 \\
\hline
\end{tabular}

(a) Correlação do item com o total do respectivo domínio, sem considerar o item no escore total.

\section{Validade}

A Tabela 2 apresenta os coeficientes de Correlação por Postos de Spearman ( $r$ ) entre os componentes do CRQ 6.0 e a EAS-40, da amostra total e para cada um dos grupos amostrais. A Tabela 3 trata da comparação dos escores do CRQ 6.0, associados aos dois grupos: G1 e G2, e indica os respectivos valores de $\mathrm{p}$.

Tabela 2

Correlação entre os escores do CRQ-6.0 e os fatores da EAS-40 para a amostra total, G1 e G2

\begin{tabular}{|c|c|c|c|c|c|c|c|c|c|}
\hline \multirow{2}{*}{ EAS-40 (a) } & \multicolumn{3}{|c|}{ Total $(\mathrm{n}=101)$} & \multicolumn{3}{|c|}{ G1 $(n=61)$} & \multicolumn{3}{|c|}{ G2 $(n=40)$} \\
\hline & D & RO & RE & D & RO & RE & D & RO & $\mathbf{R E}$ \\
\hline F1 & $0,30^{*}$ & $0,32 *$ & $0,46^{*}$ & $0,39^{*}$ & 0,23 & $0,44^{*}$ & 0,13 & $0,51 *$ & $0,57 *$ \\
\hline $\mathrm{F} 2$ & 0,17 & $0,25^{*}$ & $0,41^{*}$ & $0,29^{*}$ & $0,33^{*}$ & $0,48^{*}$ & $-0,03$ & 0,16 & $0,32 *$ \\
\hline F3 & 0,06 & $0,22 *$ & $0,30^{*}$ & 0,10 & 0,11 & $0,26^{*}$ & $-0,16$ & 0,12 & 0,21 \\
\hline F4 & $0,25^{*}$ & $0,27^{*}$ & $0,47^{*}$ & $0,27^{*}$ & $0,28^{*}$ & $0,51^{*}$ & 0,04 & 0,13 & 0,25 \\
\hline Total & 0,18 & $0,30^{*}$ & $0,47^{*}$ & $0,26^{*}$ & $0,27^{*}$ & $0,46^{*}$ & $-0,04$ & 0,25 & $0,37 *$ \\
\hline
\end{tabular}

(a) F1= Psicoticismo; F2= Obsessividade-Compulsividade; F3= Somatização; F4= Ansiedade

$*(\mathrm{p}<0,05)$ 
Tabela 3

Prova U de Mann-Whitney entre G1 e G2 de cada componente do CRQ 6.0: D, RO e RE

\begin{tabular}{|c|c|c|c|c|c|c|}
\hline \multicolumn{7}{|c|}{ Grupo controle (G2) } \\
\hline Variável CRQ & $\mathbf{N}$ & Média & DP & Mínimo & Mediana & Máximo \\
\hline $\mathrm{D}$ & 40 & 2,22 & 0,58 & 1,52 & 2,07 & 4,55 \\
\hline RO & 40 & 2,88 & 1,04 & 1,13 & 2,65 & 2,83 \\
\hline $\mathrm{RE}$ & 40 & 2,71 & 0,75 & 1,16 & 2,78 & 4,16 \\
\hline \multicolumn{7}{|c|}{ Grupo hepatite C (G1) } \\
\hline Variável CRQ & $\mathbf{N}$ & Média & DP & $\begin{array}{l}\text { Mínimo } \\
\text { Valor-p }\end{array}$ & Mediana & Máximo \\
\hline $\mathrm{D}$ & 61 & 2,43 & 0,68 & $\begin{array}{c}1,30 \\
\mathrm{p}=0,106\end{array}$ & 4,27 & 4,25 \\
\hline RO & 61 & 3,47 & 0,99 & $\begin{array}{c}1,39 \\
\mathrm{p}=0,006\end{array}$ & 3,52 & 5,83 \\
\hline $\mathrm{RE}$ & 61 & 3,13 & 0,82 & $\begin{array}{c}1,39 \\
\mathrm{p}=0,018\end{array}$ & 3,13 & 5,26 \\
\hline
\end{tabular}

Os resultados da análise comparativa entre pacientes usando medicação e pacientes sem uso da medicação são demonstrados na Tabela 4. E na Tabela 5, há a comparação de amostras de acordo com o sexo, para G1 e G2.

Tabela 4

Prova U de Mann-Whitney para participantes de G1 e G2, sem uso de medicação ( $n=40)$ e com medicação ( $n=21)$

\begin{tabular}{|c|c|c|c|c|c|c|c|}
\hline \multicolumn{8}{|c|}{ Sem uso de medicação } \\
\hline Variável CRQ & $\mathbf{N}$ & Média & DP & Mínimo & Mediana & & Máximo \\
\hline $\mathrm{D}$ & 40 & 2,37 & 0,66 & 1,30 & 2,21 & & 4,17 \\
\hline RO & 40 & 3,31 & 1,04 & 1,39 & 3,09 & & 5,83 \\
\hline $\mathrm{RE}$ & 40 & 2,99 & 0,78 & 1,47 & 3,03 & & 5,26 \\
\hline \multicolumn{8}{|c|}{ Com uso de medicação } \\
\hline Variável CRQ & $\mathbf{N}$ & Média & DP & Mínimo & Mediana & Máximo & Valor-p \\
\hline $\mathrm{D}$ & 21 & 2,54 & 0,73 & 1,30 & 2,72 & 4,25 & $\mathrm{p}=0,354$ \\
\hline RO & 21 & 3,80 & 0,82 & 2,17 & 3,78 & 5,30 & $\mathrm{p}=0,051$ \\
\hline RE & 21 & 3,41 & 0,84 & 1,79 & 3,55 & 4,68 & $\mathrm{p}=0,045$ \\
\hline
\end{tabular}

Tabela 5

Prova U de Mann-Whitney para G1 e G2, com resultados divididos pelo sexo dos participantes

\begin{tabular}{cccccccccc}
\hline Grupo & Gênero & Variável CRQ & N & Média & DP & Mínimo & Mediana & Máximo & Valor-p \\
\hline G2 & Feminino & D & 25 & 2,23 & 0,63 & 1,52 & 2,15 & 4,55 & $\mathrm{p}=0,823$ \\
& & RO & 25 & 2,78 & 1,04 & 1,13 & 2,61 & 4,65 & $\mathrm{p}=0,328$ \\
& & $\mathrm{RE}$ & 25 & 2,62 & 0,69 & 1,52 & 2,34 & 3,95 & $\mathrm{p}=0,335$ \\
& \multirow{4}{*}{ Masculino } & $\mathrm{D}$ & 15 & 2,21 & 0,51 & 1,67 & 1,97 & 3,25 & \\
& & $\mathrm{RO}$ & 15 & 3,05 & 1,04 & 1,78 & 2,83 & 4,83 & \\
& & $\mathrm{RE}$ & 15 & 2,86 & 0,84 & 1,16 & 2,89 & 4,46 & \\
G1 & \multirow{4}{*}{ Feminino } & $\mathrm{D}$ & 24 & 2,66 & 0,68 & 1,55 & 2,64 & 4,25 & $\mathrm{p}=0,047$ \\
& & $\mathrm{RO}$ & 24 & 3,79 & 1,09 & 1,87 & 3,87 & 5,73 & $\mathrm{p}=0,070$ \\
& \multirow{3}{*}{ Masculino } & $\mathrm{RE}$ & 24 & 3,57 & 0,78 & 2,24 & 3,63 & 5,26 & $\mathrm{p}=0,001$ \\
& & $\mathrm{D}$ & 37 & 2,28 & 0,65 & 1,30 & 2,12 & 3,70 & \\
& & $\mathrm{RO}$ & 37 & 3,27 & 0,88 & 1,39 & 3,17 & 5,17 & \\
\hline
\end{tabular}




\section{Discussão}

Este primeiro estudo da versão em português do CRQ 6.0 apontou resultados bastante encorajadores no que concerne às suas propriedades psicométricas para pacientes com HVC crônica, em atendimento ambulatorial. O CRQ 6.0 demonstrou consistência interna aceitável (Alfas de Cronbach $>0,80$ ) para todos os componentes: D, RO e RE (Tabela 1), corroborando os dados encontrados na literatura (Barber e cols., 1998, Weinryb e cols., 2000). Retirados alguns itens como, por exemplo, o D7, RO10 e RE8 (amostra total), o D36, RO19 e RE26 (G1) e o D7, RO10 e RE19 (G2), as consistências internas dos respectivos componentes mostraramse ainda mais altas, sugerindo que a contribuição de cada item para o fator deve ser mais bem compreendida. Para isto, pesquisas envolvendo amostras maiores e mais representativas dos pacientes com HVC (de diferentes partes do país e atendidos por diferentes instituições) devem ser realizadas, de forma a permitir outras análises estatísticas. Por ora, pode-se dizer que a versão em português do CRQ 6.0 atende às expectativas teóricas quanto à consistência interna de seus itens.

Em relação ao estudo de validade convergente (Tabela 2), quando se comparam os resultados dos dois grupos, é possível verificar que no $\mathrm{G} 1$ a associação entre a intensidade do conflito nos três componentes do CRQ 6.0 e a presença de sintomas tendem a ser muito mais evidentes, com associações significantes entre eles, especialmente quando se considera o escore total da EAS-40 (Tabela 2). Além disto, os resultados corroboraram os de outros estudos que também encontraram relação positiva entre maior conflito no relacionamento e a presença de sintomas psicopatológicos em pacientes com diagnóstico de hepatite C crônica (Miyazaki e cols., 2005; Mulder e cols., 2000; Thomson \& Finch, 2005).

Examinando especificamente as associações de cada componente do CRQ 6.0 e as dimensões da EAS-40, verifica-se que, no caso de G1, apenas a dimensão somatização (F3) da EAS-40 não apresentou associação significante com o componente $\mathrm{D}$ e que, em G2, conforme o esperado, as correlações ficaram próximas de zero. No que respeita à dimensão somatização, seus itens avaliam a presença de sintomas comuns aos transtornos somáticos, tais como: fraqueza ou tontura, dores no peito, dores nas costas ou nos quadris, náuseas, enjoos ou estômago ruim, dores musculares, problemas para respirar, ondas de calor ou frio, dormência ou formigamento em partes do corpo, fraqueza em partes do corpo e peso nos braços e pernas, entre outros (Laloni, 2001). Como o G1 foi composto por pacientes que se encontravam em atendimento médico, muitos dos quais sob medicação, é possível aventar que os sintomas físicos encontravam-se sob controle, havendo, portanto aí a intervenção de um viés sobre os resultados. Levando-se, portanto, em consideração esta ressalva, é possível dizer que os resultados apontam para a validade do CRQ 6.0 para pacientes com HVC, com a existência de associação entre a intensidade dos sintomas psicopatológicos e os conflitos relacionados aos desejos (D), em relação ao parceiro amoroso.
No componente RO (Tabela 2), além da falta de associação com a dimensão somatização (F3) da EAS-40, também não houve associação com os sintomas da dimensão psicoticismo (F1). No caso da somatização, o mesmo raciocínio utilizado para o componente $\mathrm{D}$ pode ajudar a compreender este resultado. No entanto, em relação à dimensão psicoticismo, deve-se ter cautela na interpretação dos resultados, posto que ela reúne sintomas que indicam a presença de hostilidade, depressão e ideias paranoides (Laloni, 2001) em relação ao outro, esperados em relação às respostas do outro (RO), em situações de conflito relacional. Cabe ainda mencionar que, também, não foi encontrada associação significante entre o componente $\mathrm{RO}$ e a dimensão psicoticismo em portadores de HIV/AIDS (Massei \& Yoshida, 2009), mas tal associação foi encontrada em mulheres vítimas de violência (Silva, 2008) e portadores de doenças coronarianas (Sanches, 2009). Ou seja, os resultados discrepantes demandam novas investigações para uma compreensão mais adequada da associação, ou ausência de associação entre o componente RO e a dimensão psicoticismo da EAS-40.

Quanto ao componente RE, as correlações positivas, em relação a todas as dimensões da EAS-40 no G1 (Tabela 2), falam a favor da validade deste componente. Parece que frente às frustrações decorrentes das respostas do outro, ou às expectativas de respostas do outro que não preenchem as necessidades do indivíduo, este tende a reagir com comportamentos inadequados e usualmente associados a sintomas psicopatológicos, tais como isolamento social, instabilidade emocional, ansiedade, ações e pensamentos repetidos, o que por sua vez deve agravar o conflito no relacionamento com o parceiro amoroso.

Quanto ao estudo de validade discriminante, foram encontradas diferenças para os componentes RO e RE do CRQ 6.0 (Tabela 3), com G1 apresentando maior intensidade nos conflitos amorosos, quando comparados a pessoas que não apresentam a doença. A diferença é especialmente evidente no que respeita à forma como os portadores de HVC interpretam as respostas do parceiro frente às suas necessidades e desejos (RO) e em relação às respostas que eles dão a elas (RE), nos piores momentos do relacionamento. Na comparação dos participantes com e sem medicação (Tabela 4), apenas no componente RE houve diferença significante, sugerindo que pacientes com hepatite $\mathrm{C}$ que se encontram sob medicação tendem a apresentar mais conflitos relacionados à dimensão Respostas do Eu (RE), nos conflitos amorosos. É possível que a medicação funcione como fator de alteração do humor, como sugerem os achados de Kraus e cols. (2003) que indicam que o uso da medicação em pacientes portadores de hepatite $\mathrm{C}$ crônica ocasiona maiores índices de raiva/ hostilidade, ou os de Strader e cols. (2004) que descrevem alterações de humor e irritação devido ao uso da medicação nestes pacientes. E, por consequência, haveria um aumento do conflito no componente RE, com respostas ainda mais vigorosas do indivíduo frente às frustrações de suas necessidades. Estas ilações necessitam, todavia, ser ratificadas em pesquisas futuras, em que grupos com diferentes tempos de 
medicação possam ser comparados. E quando os participantes foram divididos em função do sexo (Tabela 5), não foram observadas diferenças em nenhum dos componentes do CRQ 6.0 do G2, mas as mulheres no G1 apresentaram escores superiores aos homens nos componentes D e RE (Tabela 3), sugerindo, portanto, maior nível de conflito destas em relação aos desejos e também em relação às respostas que apresentam por não terem suas expectativas preenchidas por seus parceiros amorosos. Os resultados apontaram, portanto, para o esperado e corroboram as observações de Fabris e cols. (2006) que apontam para maiores prejuízos psicológicos entre pacientes com hepatite $\mathrm{C}$ crônica do sexo feminino.

\section{Considerações finais}

Em que pesem os limites desta pesquisa, em especial a falta de aleatoriedade na composição das amostras e o fato dos dados terem sido colhidos em apenas uma instituição, é possível dizer, a título de conclusões provisórias, que o CRQ 6.0 mostrou-se promissor na avaliação de conflitos no relacionamento amoroso de portadores de hepatite C. E, mais especificamente, que as Respostas do Eu (RE) parecem ser afetadas, nestes casos, com o agravamento dos conflitos. Isto é, pacientes com hepatite $\mathrm{C}$ crônica parecem tender a responder de forma negativa e intensa às frustrações vividas, quando sentem que as respostas do parceiro amoroso não correspondem às suas expectativas. Sendo que esta condição é especialmente observada em pacientes do sexo feminino ou que estejam fazendo uso de medicação. A identificação do grau de generalização desses resultados depende, naturalmente, de novos estudos.

\section{Referências}

Abreu-Rodrigues, M., \& Seidl, E. M. F. (2008). A importância do apoio social em pacientes coronarianos. Revista Paidéia: Cadernos de Psicologia e Educação, 18(40), 279-288.

Amódio, P., De Toni, E. N., Cavalletto, L., Mapelli, D., Bernardinello, E., Del Picolo, F., Bergamelli, C., Constanzo, R., Bergamaschi, F., Poma, S.Z., Chemello, L., Gatta, A., \& Perini, G. (2005). Mood, cognition and E.E.G. changes during interferon alpha (alpha-IFN) treatment for chronic hepatitis C. Journal Affective Disorder, 84(1), 93-98.

Barber, J. P. (1997). Central Relationship Questionnaire-6.0 (CRQ-6.0). Recuperado em 12 novembro 2008, de http:// www.med.upenn.edu/cpr/documents/CRQ6P0.pdf.

Barber, J. P., Foltz, C., De Rubeis, R. J., \& Landis, R. J. (2002). Consistency of interpersonal themes in narratives about relationships. Psychotherapy Research, 12, 139158.

Barber, J. P., Foltz, C., \& Weinryb, R. M. (1998). The Central Relationship Questionnaire: initial report. Journal of Counseling Psychology, 45(2), 131-142.
Blasiole, J. A., Shinkunas, L., LaBrecque, D. R., Arnold, R. M., \& Zickmund, S. L. (2006). Mental and physical symptoms associated with lower social support for patients with hepatitis C. World Journal of Gastroenterology, 12(29), 4665-4672.

Derogatis, L. R. (1994). Symptom Checklist-90-R (SCL90-R): administration, scoring and procedures manual (3a ed.). Minnepolis: National Computer Sistems.

Fabris, P., Tositti, G., Giordani, M. T., Baldo, V., Grasso, A., Pignattari, E., Canton, S., Rossato, S., \& Floreani, A. (2006). Assessing patients' understanding of hepatitis C virus infection and its impact on their lifestyle. Alimentary Pharmacology \& Therapeutics, 23(8), 1161-1170.

Kraus, M. R., Schäfer, A., Faller, H., Csef, H., \& Scheurlen, M. (2003). Psychiatric symptoms in patients with chronic hepatitis $\mathrm{C}$ receiving interferon alfa-2b therapy. Journal Clinical Psychiatry, 64(6), 708-714.

Laloni, D. T. (2001). Escala de Avaliação de Sintomas90-R-SCL-90-R: Adaptação, precisão e validade. Tese de Doutorado não publicada, Pontifícia Universidade Católica de Campinas, SP. Disponível em: www. bibliotecadigital.puc-campinas.edu.br/tde_busca/ arquivo.php?codArquivo=294.

Luborsky, L. (1984). Principles of psychoanalytic psychotherapy: A manual for supportive-expressive treatment. New York: Basic Books.

Luborsky, L., \& Crits-Christoph, P. (1998). Understanding transference:Tthe core conflictual relationship theme method (2a ed.). Washington, DC: American Psychological Association.

Massei, A. C., \& Yoshida, E. M. P.(2009). Avaliação das propriedades psicométricas do Questionário de Relacionamento Central 6.0 (CRQ 6.0) em portadores de HIV/AIDS. Psicologia: Teoria e Prática, 11(2) (no prelo).

McCarthy, K. S., Gibbons, M. B. C., \& Barber, J. P. (2008). The relation of rigidity across relationships with symptoms and functioning: An investigation with the revised Central Relationship Questionnaire. Journal of Counseling Psychology, 55(3), 346-358.

Ministério da Saúde (2007). De A a Z: Hepatite C. Recuperado em 7 outubro 2008, de http://portal.saude.gov.br/saude/.

Miyazack, M. C. O. S., Domingos, N. A. M., Valerio, N. I., Souza, E. F., \& Silva, R. C. M. A. (2005). Tratamento da Hepatite C: Sintomas psicológicos e estratégias de enfrentamento. Revista Brasileira de Terapia Cognitiva, 1(1), 119-128.

Mulder, R. T., Ang, M., Chapman, B., Ross, A., Steven, I.F., \& Edgar, C. (2000). Interferon treatment for Chronic Hepatitis C. Journal of Gastroenterology and Hepatology, 15(3), 300-303.

Pregnolato, A. P. F. (2005). Alexitimia e sintomas psicopatológicos em pacientes com insuficiência renal crônica. Dissertação de Mestrado não publicada, Pontifícia Universidade Católica de Campinas, Campinas, SP. Disponível em: www.bibliotecadigital.puc-campinas. edu.br/tde_busca/arquivo.php?codArquivo=251. 
Rocha, G. M. A. (2004). O Método do Tema Central de Relacionamento Conflituoso - CCRT. In E. M. P. Yoshida \& M. L. E. Enéas (Orgs.), Psicoterapias psicodinâmicas breves: Propostas atuais (pp. 69-93). Campinas: Alínea.

Rocha, G. M. A. (2007). Tradução e adaptação cultural do Central Relationship Questionnaire - CRQ. Projeto de Pesquisa com cadastro no SISNEP sob o $\mathrm{n}^{\circ}$ 0015.0.272.000-07. (manuscrito)

Sanches, F. M. (2009). Questionário de Relacionamento Central (CRQ): Evidências de validade em pacientes cardíacos. Dissertação de Mestrado não publicada, Pontifícia Universidade Católica de Campinas, Campinas, SP. Disponível em: www.bibliotecadigital.puc-campinas. edu.br/tde_busca/arquivo.php?codArquivo=490

Schutte, N. S., \& Malouff (1995). Sourcebook of adult assessment strategies.New York: Plenum Press.

Silva, F.R.C.S. (2008). Validade do Central Relationship Questionnaire - CRQ 6.0 com mulheres vítimas de violência. Dissertação de Mestrado não publicada, Pontifícia Universidade Católica de Campinas, Campinas, SP. Disponível em: www.bibliotecadigital.puc-campinas. edu.br/tde_busca/arquivo.php?codArquivo $=453$.

Strader, D. B., Wright T., Thomas, D. L., \& Seef, L. B. (2004). American Association for the Study of Liver Diseases: Diagnosis, management, and treatment of hepatitis C. Hepatology, 39, 1147-1171.

Teixeira, R., Martins-Filho, O. A., \& Oliveira, G. C. (2005). Hepatite C: Aspectos clínicos de uma epidemia silenciosa. (1a ed.). Belo Horizonte: Cooperativa Editora e de Cultura Médica - Coopmed.

Tombolato, M. C. R. (2005). Qualidade de vida e Sintomas PsicopatológicosdoEstudanteUniversitário Trabalhador. Dissertação de Mestrado não publicada, Pontifícia Universidade Católica de Campinas, Campinas, SP.

Thomson, B. J., \& Finch, R. G. (2005). Hepatitis C virus infection. Clinical Microbiological and Infection, 11(2), 86-94.

Veronesi, R., \& Focaccia, R. (2005). Tratado de infectologia. (3a ed.). São Paulo: Atheneu.

Weinryb, M. R., Barber, J. P., Foltz, C., Göransson S. G. M., \& Gustavsson, J. P. (2000). The Central Relationship Questionnaire (CRQ): Psychometric properties in a Swedish sample and cross-cultural Studies. Journal of Psychoterapy Practice and Research, 9, 201-212.

Wiseman, H., Barber J. P., Raz, A., Yam, I., Foltz, C. \& LivneSnir, S. (2002). Parental communication of Holocaust experiences and interpersonal patterns in offspring of Holocaust survivors. International Journal of Behavioral Development, 26(4), 371-381.

Yoshida, E. M. P. (2008). Significância clínica de mudança em processo de psicoterapia psicodinâmica breve. Paidéia (Ribeirão Preto), 18(40), 305-316.
Yoshida, E. M. P., \& Rocha, G. M. A, (2007). Avaliação em psicoterapia psicodinâmica. In J. C. Alchieri (Org.), Avaliação Psicológica: Perspectivas e contextos (pp. 237-288). São Paulo: Vetor.

Yoshida, E. M. P., \& Silva, F. R. C. S. (2007). Escala de Avaliação de Sintomas - 40 (EAS-40): validade e precisão em amostra não-clínica. Revista Semestral da Associação Brasileira de Psicologia Escolar e Educacional, 11(1), 89-99.

Gustavo Risso é Mestre em Psicologia pelo Programa de Pós-graduação Stricto Sensu em Psicologia da Pontifícia Universidade Católica de Campinas, bolsista CNPq.

Elisa Medici Pizão Yoshida é Professora Titular do Programa de Pós-graduação Stricto Sensu em Psicologia da Pontifícia Universidade Católica de Campinas, campus II.

Recebido: 19/12/2008

$1^{a}$ revisão: 20/05/2009

$2^{a}$ revisão: 19/11/2009

Aceite final: 02/12/2009 